\title{
Hermite-Hadamard Type Inequalities via Generalized Harmonic Exponential Convexity and Applications
}

\author{
Saad Ihsan Butt $\left(\mathbb{D},{ }^{1}\right.$ Muhammad Tariq, ${ }^{1}$ Adnan Aslam $\mathbb{D}^{2},{ }^{2}$ Hijaz Ahmad $\mathbb{D},{ }^{3}$ \\ and Taher A. Nofal ${ }^{4}$ \\ ${ }^{1}$ COMSATS University Islamabad, Lahore Campus, Pakistan \\ ${ }^{2}$ Department of Natural Sciences and Humanities, University of Engineering and Technology, Lahore (RCET), Pakistan \\ ${ }^{3}$ Department of Basic Sciences, University of Engineering and Technology, Peshawar, Pakistan \\ ${ }^{4}$ Department of Mathematic, College of Science, Taif University, P.O. Box 11099, Taif 21944, Saudi Arabia
}

Correspondence should be addressed to Hijaz Ahmad; hijaz555@gmail.com

Received 5 January 2021; Revised 23 January 2021; Accepted 31 January 2021; Published 12 February 2021

Academic Editor: Gangadharan Murugusundaramoorthy

Copyright $\odot 2021$ Saad Ihsan Butt et al. This is an open access article distributed under the Creative Commons Attribution License, which permits unrestricted use, distribution, and reproduction in any medium, provided the original work is properly cited.

In this work, we introduce the idea and concept of $m$-polynomial $p$-harmonic exponential type convex functions. In addition, we elaborate the newly introduced idea by examples and some interesting algebraic properties. As a result, several new integral inequalities are established. Finally, we investigate some applications for means. The amazing techniques and wonderful ideas of this work may excite and motivate for further activities and research in the different areas of science.

\section{Introduction}

Theory of convexity present an active, fascinating, and attractive field of research and also played prominence and amazing act in different fields of science, namely, mathematical analysis, optimization, economics, finance, engineering, management science, and game theory. Many researchers endeavor, attempt, and maintain his work on the concept of convex functions and extend and generalize its variant forms in different ways using innovative ideas and fruitful techniques. Convexity theory provides us with a unified framework to develop highly efficient, interesting, and powerful numerical techniques to tackle and to solve a wide class of problems which arise in pure and applied sciences. In recent years, the concept of convexity has been improved, generalized, and extended in many directions. The concept of convex functions also played prominence and meaningful act in the advancement of the theory of inequalities. A number of studies have shown that the theory of convex functions has a close relationship with the theory of inequalities.

The integral inequalities are useful in optimization theory, functional analysis, physics, and statistical theory. In diverse and opponent research, inequalities have a lot of applications in statistical problems, probability, and numerical quadrature formulas [1-3]. So eventually due to many generalizations, variants, extensions, widespread views, and applications, convex analysis and inequalities have become an attractive, interesting, and absorbing field for the researchers and for attention; the reader can refer to [4-6]. Recently Kadakal and Iscan [7] introduced a generalized form of convexity, namely, $n$-polynomial convex functions.

It is well known that the harmonic mean is the special case of power mean. It is often used for the situations when the average rates is desired and have a lot of applications in different field of sciences which are statistics, computer science, trigonometry, geometry, probability, finance, and electric circuit theory. Harmonic mean is the most appropriate measure for rates and ratios because it equalizes the weights of each data point. Harmonic mean is used to define the harmonic convex set. In 2003, first time harmonic convex set was introduced by Shi [8]. Harmonic and $p$-harmonic convex function was for the first time introduced and discussed by Anderson et al. [9] and Noor et al. [10], respectively. Awan et al. [11] keeping his work on generalizations, 
introduced a new class called $n$-polynomial harmonically convex function. Motivated and inspired by the ongoing activities and research in the convex analysis field, we found out that there exists a special class of function known as exponential convex function, and nowadays, a lot of people working are in this field $[12,13]$. Dragomir [14] introduced the class of exponential type convexity. After Dragomir, Awan et al. [15] studied and investigated a new class of exponentially convex functions. Kadakal and İşcan introduced a new definition of exponential type convexity in [16]. Recently, Geo et al. [17] introduced n-polynomial harmonic exponential type convex functions. The fruitful benefits and applications of exponential type convexity is used to manipulate for statistical learning, information sciences, data mining, stochastic optimization and sequential prediction [7, $18,19]$ and the references therein. Before we start, we need the following necessary known definitions and literature.

\section{Preliminaries}

In this section, we recall some known concepts.

Definition 1 (see [5]). Let $\psi: I \longrightarrow \mathbb{R}$ be a real valued function. A function $\psi$ is said to be convex, if

$$
\psi\left(\kappa \wp_{1}+(1-\kappa) \wp_{2}\right) \leq \kappa \psi\left(\wp_{1}\right)+(1-\kappa) \psi\left(\wp_{2}\right),
$$

holds for all $\wp_{1}, \wp_{2} \in I$ and $\kappa \in[0,1]$.

Definition 2 (see [20]). A function $\psi: I \subseteq(0, \infty) \longrightarrow \mathbb{R}$ is said to be harmonic convex, if

$$
\psi\left(\frac{\wp_{1} \wp_{2}}{\kappa \wp_{2}+(1-\kappa) \wp_{1}}\right) \leq \kappa \psi\left(\wp_{1}\right)+(1-\kappa) \psi\left(\wp_{2}\right),
$$

holds for all $\wp_{1}, \wp_{2} \in I$ and $\kappa \in[0,1]$.

For the harmonic convex function, İşcan [20] provided the Hermite-Hadamard type inequality.

Definition 3 (see [21]). A function $\psi: I \longrightarrow \mathbb{R}$ is said to be $p-$ harmonic convex, if

$$
\psi\left(\left[\frac{\wp_{1}^{p} \wp_{2}^{p}}{\kappa \wp_{2}^{p}+(1-\kappa) \wp_{1}^{p}}\right]^{1 / p}\right) \leq \kappa \psi\left(\wp_{1}\right)+(1-\kappa) \psi\left(\wp_{2}\right),
$$

holds for all $\wp_{1}, \wp_{2} \in I$ and $\kappa \in[0,1]$.

Note that $\kappa=1 / 2$ in (3), we get the following inequality:

$$
\psi\left(\left[\frac{2 \wp_{1}^{p} \wp_{2}^{p}}{\wp_{1}^{p}+\wp_{2}^{p}}\right]^{1 / p}\right) \leq \frac{\psi\left(\wp_{1}\right)+\psi\left(\wp_{2}\right)}{2},
$$

holds for all $\wp_{1}, \wp_{2} \in I$.

The function $\psi$ is called Jensen $p$-harmonic convex function.
If we put $p=-1$ and $p=1$, then $p$-harmonic convex sets and $p$-harmonic convex functions collapse to classical convex sets, harmonic convex sets, and harmonic convex functions, respectively.

Definition 4 (see [17]). A function $\psi: I \subseteq(0,+\infty) \longrightarrow[0,+$ $\infty)$ is called $m$-polynomial harmonic exponential type convex function, if

$$
\begin{aligned}
\psi\left(\frac{\wp_{1} \wp_{2}}{\kappa \wp_{2}+(1-\kappa) \wp_{1}}\right) \leq & \frac{1}{m} \sum_{j=1}^{m}\left(e^{\kappa}-1\right)^{j} \psi\left(\wp_{1}\right) \\
& +\frac{1}{m} \sum_{j=1}^{m}\left(e^{1-\kappa}-1\right)^{j} \psi\left(\wp_{2}\right),
\end{aligned}
$$

holds for every $\wp_{1}, \wp_{2} \in I, m \in \mathbb{N}$ and $\kappa \in[0,1]$.

Motivated by the above results, literature, and ongoing activities and research, we organise the paper in the following way. Firstly, we will give the idea and its algebraic properties of $m$-polynomial $p$-harmonic exponential type convex functions. Secondly, we will derive the new sort of $(\mathrm{H}-\mathrm{H})$ and refinements of $(\mathrm{H}-\mathrm{H})$ type inequalities by using the newly introduced idea. Finally, we will give some applications for means and conclusion.

\section{Generalized Exponential Type Convex Functions and Its Properties}

We are going to introduce a new definition called $m$-polynomial $p$-harmonic exponential type convex function and will study some of their algebraic properties. Throughout the paper, one thing gets in mind $m$ represents finite $\mathbb{Z}^{+}, m$-poly $p$-harmonic exp convex function represents $m$-polynomial $p$ -harmonic exponential type convex function and $(\mathrm{H}-\mathrm{H})$ represents Hermite-Hadamard.

Definition 5. A function $\psi: I \subseteq(0,+\infty) \longrightarrow[0,+\infty)$ is called $m$-poly $p$-harmonic exp convex, if

$$
\begin{aligned}
\psi\left(\left[\frac{\wp_{1}^{p} \wp_{2}^{p}}{\kappa \wp_{2}^{p}+(1-\kappa) \wp_{1}^{p}}\right]^{1 / p}\right) \leq & \frac{1}{m} \sum_{j=1}^{m}\left(e^{\kappa}-1\right)^{j} \psi\left(\wp_{1}\right) \\
& +\frac{1}{m} \sum_{j=1}^{m}\left(e^{1-\kappa}-1\right)^{j} \psi\left(\wp_{2}\right),
\end{aligned}
$$

holds for every $\wp_{1}, \wp_{2} \in I, m \in \mathbb{N}$ and $\kappa \in[0,1]$.

\section{Remark 6.}

(i) Taking $m=1$ in Definition 5, we obtain the following new definition about $p$-harmonically exp convex function: 
$\psi\left(\left[\frac{\wp_{1}^{p} \wp_{2}^{p}}{\kappa \wp_{2}^{p}+(1-\kappa) \wp_{1}^{p}}\right]^{1 / p}\right) \leq\left(e^{\kappa}-1\right) \psi\left(\wp_{1}\right)+\left(e^{1-\kappa}-1\right) \psi\left(\wp_{2}\right)$

(ii) Taking $m=2$ in Definition 5, we obtain the following new definition about 2 -poly $p$-harmonically exp convex function:

$\psi\left(\left[\frac{\wp_{1}^{p} \wp_{2}^{p}}{\kappa \wp_{2}^{p}+(1-\kappa) \wp_{1}^{p}}\right]^{1 / p}\right) \leq\left(\frac{e^{2 \kappa}-e^{\kappa}}{2}\right) \psi\left(\wp_{1}\right)+\left(\frac{e^{2(1-\kappa)}-e^{1-\kappa}}{2}\right) \psi\left(\wp_{2}\right)$

(iii) Taking $p=1$ in Definition 5, then, we get a definition, namely, $m$-poly harmonically exp convex function which is defined by Geo et al. [17]

(iv) Taking $p=-1$ in Definition 5, we obtain the following new definition about $m$-poly exp convex function:

$\psi\left(\kappa \wp_{1}+(1-\kappa) \wp_{2}\right) \leq \frac{1}{m} \sum_{j=1}^{m}\left(e^{\kappa}-1\right)^{j} \psi\left(\wp_{1}\right)+\frac{1}{m} \sum_{j=1}^{m}\left(e^{1-\kappa}-1\right)^{j} \psi\left(\wp_{2}\right)$

(v) Taking $m=1$ and $p=1$ in Definition 5, we obtain the following new definition about harmonically exp type convex function:

$$
\psi\left(\frac{\wp_{1} \wp_{2}}{\kappa \wp_{2}+(1-\kappa) \wp_{1}}\right) \leq\left(e^{\kappa}-1\right) \psi\left(\wp_{1}\right)+\left(e^{1-\kappa}-1\right) \psi\left(\wp_{2}\right)
$$

(vi) Taking $m=1$ and $p=-1$ in Definition 5 , then, we get a definition, namely, exponential type convex function which is defined by Kadakal et al. [16]

That is the beauty of this newly introduce definition if we put the values of $m$ and $p$, then, we obtain new inequalities and also found some results which connect with previous results.

Lemma 7. The following inequalities $(1 / m) \sum_{j=1}^{m}\left(e^{\kappa}-1\right)^{j} \geq \kappa$ and $(1 / m) \sum_{j=1}^{m}\left(e^{(1-\kappa)}-1\right)^{j} \geq(1-\kappa)$ are hold. If for all $\kappa \in$ $[0,1]$.

Proof. The rest of the proof is clearly seen.

Proposition 8. Every p-harmonic convex function is m-poly p-harmonic exp convex function.
Proof. Using the definition of $p$-harmonic convex function and from the Lemma 7 , since $\kappa \leq(1 / m) \sum_{j=1}^{m}\left(e^{\kappa}-1\right)^{j}$ and $(1-\kappa) \leq(1 / m) \sum_{j=1}^{m}\left(e^{1-\kappa}-1\right)^{j}$ for all $\kappa \in[0,1]$, we have

$$
\begin{aligned}
\psi\left(\left[\frac{\wp_{1}^{p} \wp_{2}^{p}}{\kappa \wp_{2}^{p}+(1-\kappa) \wp_{1}^{p}}\right]^{1 / p}\right) \leq & \kappa \psi\left(\wp_{1}\right)+(1-\kappa) \psi\left(\wp_{2}\right) \\
\leq & \frac{1}{m} \sum_{j=1}^{m}\left(e^{\kappa}-1\right)^{j} \psi\left(\wp_{1}\right) \\
& +\frac{1}{m} \sum_{j=1}^{m}\left(e^{1-\kappa}-1\right)^{j} \psi\left(\wp_{2}\right) .
\end{aligned}
$$

Proposition 9. Every $m$-poly p-harmonic exp convex function is p-harmonic h-convex function with $h(\kappa)=1 / m$ $\sum_{j=1}^{m}\left(e^{\kappa}-1\right)^{j}$.

Proof.

$$
\begin{aligned}
\psi\left(\left[\frac{\wp_{1}^{p} \wp_{2}^{p}}{\kappa \wp_{2}^{p}+(1-\kappa) \wp_{1}^{p}}\right]^{1 / p}\right) \leq & \frac{1}{m} \sum_{j=1}^{m}\left(e^{\kappa}-1\right)^{j} \psi\left(\wp_{1}\right) \\
& +\frac{1}{m} \sum_{j=1}^{m}\left(e^{1-\kappa}-1\right)^{j} \psi\left(\wp_{2}\right) \\
\leq & h(\kappa) \psi\left(\wp_{1}\right)+h(1-\kappa) \psi\left(\wp_{2}\right) .
\end{aligned}
$$

Remark 10.

(i) If $p=1$ in Proposition 9, then as a result, we get harmonically convex function, which is introduced by Noor et al. [22]

(ii) If $p=-1$ in Proposition 9, then as a result, we get $h-$ convex function, which is defined by Varošanec [6]

Now, we make and investigate some examples by way of newly introduced definition.

Example 11. If $\psi(v)=v^{p+1}, \forall x \in(0, \infty)$ is $p$-harmonic convex function, then according to Proposition 8 , it is an $m$-poly $p$-harmonic exp convex function.

Example 12. If $\psi(v)=1 / v^{2 p}, \forall x \in \mathbb{R} \backslash\{0\}$ is $p$-harmonic convex function, then according to Proposition 8 , it is an $m$-poly $p$-harmonic exp convex function.

Now, we will discuss and investigate some of its algebraic properties.

Theorem 13. Let $\psi, \varphi:\left[\wp_{1}, \wp_{2}\right] \longrightarrow \mathbb{R}$. If $\psi$ and $\varphi$ are two $m-$ poly p-harmonic exp convex functions, then

(i) $\psi+\varphi$ is an m-poly p-harmonic exp convex function 
(ii) For $c \in \mathbb{R}(c \geq 0)$, $c \psi$ is an m-poly p-harmonic exp convex function

Proof.

(i) Let $\psi$ and $\varphi$ be an $m$-poly $p$-harmonic exp convex, then

$$
\begin{aligned}
(\psi+\varphi)( & {\left.\left[\frac{\wp_{1}^{p} \wp_{2}^{p}}{\kappa \wp_{2}^{p}+(1-\kappa) \wp_{1}^{p}}\right]^{1 / p}\right) } \\
= & \psi\left(\left[\frac{\wp_{1}^{p} \wp_{2}^{p}}{\kappa \wp_{2}^{p}+(1-\kappa) \wp_{1}^{p}}\right]^{1 / p}\right)+\varphi\left(\left[\frac{\wp_{1}^{p} \wp_{2}^{p}}{\kappa \wp_{2}^{p}+(1-\kappa) \wp_{1}^{p}}\right]^{1 / p}\right) \\
\leq & \frac{1}{m} \sum_{j=1}^{m}\left(e^{\kappa}-1\right)^{j} \psi\left(\wp_{1}\right)+\frac{1}{m} \sum_{j=1}^{m}\left(e^{1-\kappa}-1\right)^{j} \psi\left(\wp_{2}\right) \\
& +\frac{1}{m} \sum_{j=1}^{m}\left(e^{\kappa}-1\right)^{j} \varphi\left(\wp_{1}\right)+\frac{1}{m} \sum_{j=1}^{m}\left(e^{1-\kappa}-1\right)^{j} \varphi\left(\wp_{2}\right) \\
= & \frac{1}{m} \sum_{j=1}^{m}\left(e^{\kappa}-1\right)^{j}\left[\psi\left(\wp_{1}\right)+\varphi\left(\wp_{1}\right)\right] \\
& +\frac{1}{m} \sum_{j=1}^{m}\left(e^{1-\kappa}-1\right)^{j}\left[\psi\left(\wp_{2}\right)+\varphi\left(\wp_{2}\right)\right] \\
= & \frac{1}{m} \sum_{j=1}^{m}\left(e^{\kappa}-1\right)^{j}(\psi+\varphi)\left(\wp_{1}\right)+\frac{1}{m} \sum_{j=1}^{m}\left(e^{1-\kappa}-1\right)^{j}(\psi+\varphi)\left(\wp_{2}\right)
\end{aligned}
$$

(ii) Let $\psi$ be an $m$-pol $p$-harmonic exp convex, then

$$
\begin{aligned}
& (c \psi)\left(\left[\frac{\wp_{1}^{p} \wp_{2}^{p}}{\kappa \wp_{2}^{p}+(1-\kappa) \wp_{1}^{p}}\right]^{1 / p}\right) \\
& \quad \leq c\left[\frac{1}{m} \sum_{j=1}^{m}\left(e^{\kappa}-1\right)^{j} \psi\left(\wp_{1}\right)+\frac{1}{m} \sum_{j=1}^{m}\left(e^{1-\kappa}-1\right)^{j} \psi\left(\wp_{2}\right)\right] \\
& \quad=\frac{1}{m} \sum_{j=1}^{m}\left(e^{\kappa}-1\right)^{j} c \psi\left(\wp_{1}\right)+\frac{1}{m} \sum_{j=1}^{m}\left(e^{1-\kappa}-1\right)^{j} c \psi\left(\wp_{2}\right) \\
& \quad=\frac{1}{m} \sum_{j=1}^{m}\left(e^{\kappa}-1\right)^{j}(c \psi)\left(\wp_{1}\right)+\frac{1}{m} \sum_{j=1}^{m}\left(e^{1-\kappa}-1\right)^{j}(c \psi)\left(\wp_{2}\right)
\end{aligned}
$$

which completes the proof.

\section{Remark 14}

(i) If $m=1$ in Theorem 13, then as a result, we get the $\psi+\varphi$ and $c \psi$ are $p$-harmonic exp convex functions

(ii) If $p=1$ in Theorem 13, then as a result, we get Theorem 3.2 in [17]

(iii) If $m=p=1$ in Theorem 13, then as a result, we get the $\psi+\varphi$ and $c \psi$ are harmonic exp convex functions (iv) If $p=-1$ in Theorem 13, then as a result, we get the $\psi+\varphi$ and $c \psi$ are $m$-poly exp convex functions

(v) If $m=1$ and $p=-1$ in Theorem 13, then as a result, we get Theorem 2.1 in [16]

Theorem 15. Let $\psi: I=\left[\wp_{1}, \wp_{2}\right] \longrightarrow J$ be $p$-harmonic convex function and $\varphi: J \longrightarrow \mathbb{R}$ is nondecreasing and $m$-poly exp convex function. Then, the function $\varphi \circ \psi: I=\left[\wp_{1}, \wp_{2}\right] \longrightarrow$ $\mathbb{R}$ is an $m$-poly p-harmonic exp convex function.

Proof. $\forall \wp_{1}, \wp_{2} \in I$, and $\kappa \in[0,1]$, we have

$$
\begin{aligned}
(\varphi \circ \psi) & \left(\left[\frac{\wp_{1}^{p} \wp_{2}^{p}}{\kappa \wp_{2}^{p}+(1-\kappa) \wp_{1}^{p}}\right]^{1 / p}\right)=\varphi\left(\psi\left[\frac{\wp_{1}^{p} \wp_{2}^{p}}{\kappa \wp_{2}^{p}+(1-\kappa) \wp_{1}^{p}}\right]^{1 / p}\right) \\
\leq & \varphi\left(\kappa \psi\left(\wp_{1}\right)+(1-\kappa) \psi\left(\wp_{2}\right)\right) \leq \frac{1}{m} \sum_{j=1}^{m}\left(e^{\kappa}-1\right)^{j} \varphi\left(\psi\left(\wp_{1}\right)\right) \\
& +\frac{1}{m} \sum_{j=1}^{m}\left(e^{1-\kappa}-1\right)^{j} \varphi\left(\psi\left(\wp_{2}\right)\right)=\frac{1}{m} \sum_{j=1}^{m}\left(e^{\kappa}-1\right)^{j}(\varphi \circ \psi)\left(\wp_{1}\right) \\
& +\frac{1}{m} \sum_{j=1}^{m}\left(e^{1-\kappa}-1\right)^{j}(\varphi \circ \psi)\left(\wp_{2}\right) .
\end{aligned}
$$

\section{Remark 16.}

(i) In case of $m=1$, we investigate the following new inequality:

$$
\begin{aligned}
(\varphi \circ \psi)\left(\left[\frac{\wp_{1}^{p} \wp_{2}^{p}}{t \wp_{2}^{p}+(1-t) \wp_{1}^{p}}\right]^{1 / p}\right) \leq & \left(e^{\kappa}-1\right)(\varphi \circ \psi)\left(\wp_{1}\right) \\
& +\left(e^{1-\kappa}-1\right)(\varphi \circ \psi)\left(\wp_{2}\right)
\end{aligned}
$$

(ii) In case of $p=1$, the above Theorem 15 collapses to Theorem 3.3 in [17]

(iii) In case of $m=p=1$, as a result, we obtain the following new inequality:

$$
\begin{aligned}
(\varphi \circ \psi)\left[\frac{\wp_{1} \wp_{2}}{\kappa \wp_{2}+(1-\kappa) \wp_{1}}\right] \leq & \left(e^{\kappa}-1\right)(\varphi \circ \psi)\left(\wp_{1}\right) \\
& +\left(e^{1-\kappa}-1\right)(\varphi \circ \psi)\left(\wp_{2}\right)
\end{aligned}
$$

(iv) In case of $p=-1$, then, the above Theorem 15 collapses to the following new inequality: 


$$
\begin{aligned}
(\varphi \circ \psi)\left(\kappa \wp_{1}+(1-\kappa) \wp_{2}\right) \leq & \frac{1}{m} \sum_{j=1}^{m}\left(e^{\kappa}-1\right)^{j}(\varphi \circ \psi)\left(\wp_{1}\right) \\
& +\frac{1}{m} \sum_{j=1}^{m}\left(e^{1-\kappa}-1\right)^{j}(\varphi \circ \psi)\left(\wp_{2}\right)
\end{aligned}
$$

(v) In case of $m=1$ and $p=-1$, as a result, the above Theorem 15 collapses to the Theorem (2.2) in [16]

Theorem 17. Let $0<\wp_{1}<\wp_{2}, \psi_{j}:\left[\wp_{1}, \wp_{2}\right] \longrightarrow[0,+\infty)$ be a class of $m$-poly p-harmonic exp convex functions and $\psi(u)$ $=\sup _{j} \psi_{j}(u)$. Then, $\psi$ is an $m$-poly $p$-harmonic exp convex function and $U=\left\{u \in\left[\wp_{1}, \wp_{2}\right]: \psi(u)<+\infty\right\}$ is an interval.

Proof. Let $\wp_{1}, \wp_{2} \in U$ and $\kappa \in[0,1]$, then

$$
\begin{aligned}
& \psi\left(\left[\frac{\wp_{1}^{p} \wp_{2}^{p}}{\kappa \wp_{2}^{p}+(1-\kappa) \wp_{1}^{p}}\right]^{1 / p}\right)=\sup _{j} \psi_{j}\left(\left[\frac{\wp_{1}^{p} \wp_{2}^{p}}{\kappa \wp_{2}^{p}+(1-\kappa) \wp_{1}^{p}}\right]^{1 / p}\right) \\
& \quad \leq \frac{1}{m} \sum_{j=1}^{m}\left(e^{\kappa}-1\right)^{j} \sup _{j} \psi_{j}\left(\wp_{1}\right)+\frac{1}{m} \sum_{j=1}^{m}\left(e^{1-\kappa}-1\right)^{j} \sup _{j} \psi_{j}\left(\wp_{2}\right) \\
& \quad=\frac{1}{m} \sum_{j=1}^{m}\left(e^{\kappa}-1\right)^{j} \psi\left(\wp_{1}\right)+\frac{1}{m} \sum_{j=1}^{m}\left(e^{1-\kappa}-1\right)^{j} \psi\left(\wp_{2}\right)<+\infty,
\end{aligned}
$$

which completes the proof.

\section{Remark 18.}

(i) In case of $p=1$, as a result, we get Theorem 3.4 in [17]

(ii) In case of $m=1$ and $p=-1$ in Theorem 17, as a result, we get Theorem 2.3 in [16]

Theorem 19. If $\psi:\left[\wp_{1}, \wp_{2}\right] \longrightarrow \mathbb{R}$ is an $m$-poly p-harmonic exp convex then $\psi$ is bounded on $\left[\wp_{1}, \wp_{2}\right]$.

Proof. Let $x \in\left[\wp_{1}, \wp_{2}\right]$ and $L=\max \left\{\psi\left(\wp_{1}\right), \psi\left(\wp_{2}\right)\right\}$, then, there exist $\exists \kappa \in[0,1]$ such that $x=\left[\left(\wp_{1}^{p} \wp_{2}^{p}\right) /\left(\kappa \wp_{2}^{p}+(1-\kappa)\right.\right.$ $\left.\left.\wp_{1}^{p}\right)\right]^{1 / p}$. Thus, since $e^{\kappa} \leq e$ and $e^{1-\kappa} \leq e$, we have

$$
\begin{aligned}
\psi(x)= & \psi\left(\left[\frac{\wp_{1}^{p} \wp_{2}^{p}}{\kappa \wp_{2}^{p}+(1-\kappa) \wp_{1}^{p}}\right]^{1 / p}\right) \leq \frac{1}{m} \sum_{j=1}^{m}\left(e^{\kappa}-1\right)^{j} \psi\left(\wp_{1}\right) \\
& +\frac{1}{m} \sum_{j=1}^{m}\left(e^{1-\kappa}-1\right)^{j} \psi\left(\wp_{2}\right) \leq \frac{1}{m} \sum_{j=1}^{m}\left(e^{\kappa}+e^{1-\kappa}-2\right)^{j} \cdot L \\
\leq & \frac{2 L}{m} \sum_{j=1}^{m}\left[(e-1)^{j}\right]=M .
\end{aligned}
$$

The above proof clearly shows that $\psi$ is bounded above from $M$. For bounded below, the readers using the identical concept as in Theorem 2.4 in [16].

Remark 20.

(i) In case of $p=1$, we obtain Theorem 3.5 in [17]

(ii) In case of $m=1$ and $p=-1$, we obtain Theorem 2.4 in [16]

\section{4. (H-H) Type Inequality via Generalized Exponential Type Convexity}

The main object of this section is to investigate and prove a new version of $(\mathrm{H}-\mathrm{H})$ type inequality using $m$-poly $p-$ harmonic exp convexity.

Theorem 21. Let $\psi:\left[\wp_{1}, \wp_{2}\right] \longrightarrow[0,+\infty)$ be an $m$-poly $p-$ harmonic exp convex function. If $\psi \in L\left[\wp_{1}, \wp_{2}\right]$, then

$$
\begin{aligned}
\frac{m}{2 \sum_{j=1}^{m}(\sqrt{e}-1)^{j}} \psi & \left(\left[\frac{2 \wp_{1}^{p} \wp_{2}^{p}}{\wp_{1}^{p}+\wp_{2}^{p}}\right]^{1 / p}\right) \leq \frac{p \wp_{1}^{p} \wp_{2}^{p}}{\wp_{2}^{p}-\wp_{1}^{p}} \int_{\wp_{1}}^{\wp_{2}} \frac{\psi(v)}{v^{p+1}} d v \\
& \leq\left[\frac{\psi\left(\wp_{1}\right)+\psi\left(\wp_{2}\right)}{m}\right] \sum_{j=1}^{m}[e-2]^{j}
\end{aligned}
$$

Proof. Since $\psi$ is an $m$-poly $p$-harmonic exp convex function, we have

$$
\psi\left(\left[\frac{x^{p} y^{p}}{\kappa y^{p}+(1-\kappa) x^{p}}\right]^{1 / p}\right) \leq \frac{1}{m} \sum_{j=1}^{m}\left(e^{\kappa}-1\right)^{j} \psi(x)+\frac{1}{m} \sum_{j=1}^{m}\left(e^{1-\kappa}-1\right)^{j} \psi(y),
$$

which lead to

$\psi\left(\left[\frac{2 x^{p} y^{p}}{x^{p}+y^{p}}\right]^{1 / p}\right) \leq \frac{1}{m} \sum_{j=1}^{m}(\sqrt{e}-1)^{j} \psi(x)+\frac{1}{m} \sum_{j=1}^{m}(\sqrt{e}-1)^{j} \psi(y)$.

Using the change of variables, we get

$$
\begin{aligned}
\psi\left(\left[\frac{2 \wp_{1}^{p} \wp_{2}^{p}}{\wp_{1}^{p}+\wp_{2}^{p}}\right]^{1 / p}\right) \leq & \frac{1}{m} \sum_{j=1}^{m}(\sqrt{e}-1)^{j} \times\left\{\psi\left(\left[\frac{\wp_{1}^{p} \wp_{2}^{p}}{\left(\kappa \wp_{2}^{p}+(1-\kappa) \wp_{1}^{p}\right)}\right]^{1 / p}\right)\right. \\
& \left.+\psi\left(\left[\frac{\wp_{1}^{p} \wp_{2}^{p}}{\left(\kappa \wp_{1}^{p}+(1-\kappa) \wp_{2}^{p}\right)}\right]^{1 / p}\right)\right\} .
\end{aligned}
$$

Integrating the above inequality with respect to $\kappa$ on $[0,1]$, we obtain 
$\frac{m}{2 \sum_{j=1}^{m}(\sqrt{e}-1)^{j}} \psi\left(\left[\frac{2 \wp_{1}^{p} \wp_{2}^{p}}{\wp_{1}^{p}+\wp_{2}^{p}}\right]^{1 / p}\right) \leq \frac{p \wp_{1}^{p} \wp_{2}^{p}}{\wp_{2}^{p}-\wp_{1}^{p}} \int_{\wp_{1}}^{\wp_{2}} \frac{\psi(v)}{v^{p+1}} d v$,

which completes the left side inequality.

For the right side inequality, first of all, we change the variable of integration by $v=\left[\left(\wp_{1}^{p} \wp_{2}^{p}\right) /\left(\kappa \wp_{2}^{p}+(1-\kappa) \wp_{1}^{p}\right)\right]^{1 / p}$ and using Definition 5 for the function $\psi$, we have

$$
\begin{aligned}
\frac{p \wp_{1}^{p} \wp_{2}^{p}}{\wp_{2}^{p}-\wp_{1}^{p}} \int_{\wp_{1}}^{\wp_{2}} \frac{\psi(v)}{v^{p+1}} d v= & \int_{0}^{1} \psi\left(\left[\frac{\wp_{1}^{p} \wp_{2}^{p}}{\kappa \wp_{2}^{p}+(1-\kappa) \wp_{1}^{p}}\right]^{1 / p}\right) d \kappa \\
\leq & \int_{0}^{1}\left[\frac{1}{m} \sum_{j=1}^{m}\left(e^{\kappa}-1\right)^{j} \psi\left(\wp_{1}\right)\right. \\
& \left.+\frac{1}{m} \sum_{j=1}^{m}\left(e^{1-\kappa}-1\right)^{j} \psi\left(\wp_{2}\right)\right] d \kappa \\
= & \frac{\psi\left(\wp_{1}\right)}{m} \sum_{j=1}^{m} \int_{0}^{1}\left(e^{t}-1\right)^{j} d \kappa \\
& +\frac{\psi\left(\wp_{2}\right)}{m} \sum_{j=1}^{m} \int_{0}^{1}\left(e^{1-\kappa}-1\right)^{j} d \kappa \\
= & {\left[\frac{\psi\left(\wp_{1}\right)+\psi\left(\wp_{2}\right)}{m}\right] \sum_{j=1}^{m}[e-2]^{j}, }
\end{aligned}
$$

which completes the proof.

Corollary 22. In case of $m=1$ in Theorem 21, then, we get the following new $(H-H)$ type inequality for p-harmonic exp convex functions:

$$
\begin{aligned}
\frac{1}{2(\sqrt{e}-1)} \psi\left(\left[\frac{2 \wp_{1}^{p} \wp_{2}^{p}}{\wp_{1}^{p}+\wp_{2}^{p}}\right]^{1 / p}\right) & \leq \frac{p \wp_{1}^{p} \wp_{2}^{p}}{\wp_{2}^{p}-\wp_{1}^{p}} \int_{\wp_{1}}^{\wp_{2}} \frac{\psi(v)}{v^{p+1}} d v \\
& \leq(e-2)\left[\psi\left(\wp_{1}\right)+\psi\left(\wp_{2}\right)\right] .
\end{aligned}
$$

Corollary 23. In case of $p=-1$ in Theorem 21, then as a result, we investigate the following new $(\mathrm{H}-\mathrm{H})$ type inequality for m-poly exp convex functions:

$$
\begin{aligned}
\frac{m}{2 \sum_{j=1}^{m}(\sqrt{e}-1)^{j}} \psi\left(\frac{\wp_{1}+\wp_{2}}{2}\right) & \leq \frac{1}{\wp_{2}-\wp_{1}} \int_{\wp_{1}}^{\wp_{2}} \psi(v) d v \\
& \leq\left(\frac{\psi\left(\wp_{1}\right)+\psi\left(\wp_{2}\right)}{m}\right) \sum_{j=1}^{m}[e-2]^{j} .
\end{aligned}
$$

Remark 24.

(i) In case of $\mathrm{p}=1$, then as a result, we obtain Theorem 4.1 in [17] (ii) In case of $m=1$ and $p=-1$, then as a result, we obtain Theorem 3.1 in [16]

(iii) In case of $m=1$ and $p=1$, then as a result, we obtain Corollary lin [17]

\section{Refinements of $(\mathrm{H}-\mathrm{H})$ Type Inequality via Generalized Exponential Type Convexity}

In this section, in order to prove our main results regarding on some Hermite-Hadamard type inequalities for $m$-poly $p$ -harmonic exp convex function, we need the following lemmas:

Lemma 25. Let $\psi: I=\left[\wp_{1}, \wp_{2}\right] \subseteq \mathbb{R} \backslash\{0\} \longrightarrow \mathbb{R}$ be differentiable function on the $I^{\circ}$ of I. If $\psi^{\prime} \in L\left[\wp_{1}, \wp_{2}\right]$, then

$$
\begin{aligned}
\frac{\psi\left(\wp_{1}\right)+\psi\left(\wp_{2}\right)}{2} & -\frac{p \wp_{1}^{p} \wp_{2}^{p}}{\wp_{2}^{p}-\wp_{1}^{p}} \int_{\wp_{1}}^{\wp_{2}} \frac{\psi(x)}{x^{1+p}} d x \\
& =\frac{\wp_{1} \wp_{2}\left(\wp_{2}^{p}-\wp_{1}^{p}\right)}{2 p} \int_{0}^{1} \frac{\mu(\kappa)}{A_{\kappa}^{p+1}} \psi^{\prime}\left(\frac{\wp_{1} \wp_{2}}{A_{\kappa}}\right) d \kappa,
\end{aligned}
$$

where $A_{\kappa}=\left[\kappa \wp_{2}^{p}+(1-\kappa) \wp_{1}^{p}\right]^{1 / p}$ and $\mu(\kappa)=(1-2 \kappa)$.

Proof. Let

$I=\frac{\wp_{2}^{p}-\wp_{1}^{p}}{2 p \wp_{1}^{p} \wp_{2}^{p}} \int_{0}^{1}(1-2 \kappa)\left[\frac{\wp_{1}^{p} \wp_{2}^{p}}{\kappa \wp_{2}^{p}+(1-\kappa) \wp_{1}^{p}}\right]^{1+\frac{1}{p}} \psi^{\prime}\left(\left[\frac{\wp_{1}^{p} \wp_{2}^{p}}{\kappa \wp_{2}^{p}+(1-\kappa) \wp_{1}^{p}}\right]^{1 / p}\right)$.

Using integration by parts

$$
\begin{aligned}
I= & \frac{\wp_{2}^{p}-\wp_{1}^{p}}{2 p \wp_{1}^{p} \wp_{2}^{p}}\left\{\left|\frac{-p \wp_{1}^{p} \wp_{2}^{p}}{\wp_{2}^{p}-\wp_{1}^{p}}(1-2 \kappa) \psi\left(\left[\frac{\wp_{1}^{p} \wp_{2}^{p}}{\kappa \wp_{2}^{p}+(1-\kappa) \wp_{1}^{p}}\right]^{1 / p}\right)\right|_{0}^{1}\right. \\
& \left.-\frac{2 p \wp_{1}^{p} \wp_{2}^{p}}{\wp_{2}^{p}-\wp_{1}^{p}} \int_{0}^{1} \psi\left(\left[\frac{\wp_{1}^{p} \wp_{2}^{p}}{\kappa \wp_{2}^{p}+(1-\kappa) \wp_{1}^{p}}\right]^{1 / p}\right) d \kappa\right\} \\
= & \frac{\psi\left(\wp_{1}\right)+\psi\left(\wp_{2}\right)}{2}-\frac{p \wp_{1}^{p} \wp_{2}^{p}}{\wp_{2}^{p}-\wp_{1}^{p}} \int_{\wp_{1}}^{\wp_{2}} \frac{\psi(x)}{x^{1+p}} d x .
\end{aligned}
$$

Lemma 26 (see [23]). Let $\psi: I=\left[\wp_{1}, \wp_{2}\right] \subseteq \mathbb{R} \backslash\{0\} \longrightarrow \mathbb{R}$ be differentiable function on the $I^{\circ}$ of I. If $\psi^{\prime} \in L\left[\wp_{1}, \wp_{2}\right]$, then

$$
\begin{array}{r}
\frac{1}{8}\left[\psi\left(\wp_{1}\right)+3 \psi\left(\left[\frac{3 \wp_{1}^{p} \wp_{2}^{p}}{\wp_{1}^{p}+2 \wp_{2}^{p}}\right]^{1 / p}\right)+3 \psi\left(\left[\frac{3 \wp_{1}^{p} \wp_{2}^{p}}{2 \wp_{1}^{p}+\wp_{2}^{p}}\right]^{1 / p}\right)+\psi\left(\wp_{2}\right)\right] \\
-\frac{p \wp_{1}^{p} \wp_{2}^{p}}{\wp_{2}^{p}-\wp_{1}^{p}} \int_{\wp_{1}}^{\wp_{2}} \frac{\psi(x)}{x^{1+p}} d x=\frac{\wp_{1} \wp_{2}\left(\wp_{2}^{p}-\wp_{1}^{p}\right)}{p} \int_{0}^{1} \frac{\mu(\kappa)}{A_{\kappa}^{p+1}} \psi^{\prime}\left(\frac{\wp_{1} \wp_{2}}{A_{\kappa}}\right) d \kappa,
\end{array}
$$


where $A_{\kappa}=\left[\kappa \wp_{2}^{p}+(1-\kappa) \wp_{1}^{p}\right]^{1 / p}$ and

$$
\mu(\kappa)= \begin{cases}\kappa-\frac{1}{8}, & \text { if } \kappa \in\left[0, \frac{1}{3}\right), \\ \kappa-\frac{1}{2}, & \text { if } \kappa \in\left[\frac{1}{3}, \frac{2}{3}\right), \\ \kappa-\frac{7}{8}, & \text { if } \kappa \in\left[\frac{2}{3}, 1\right) .\end{cases}
$$

Theorem 27. Let $\psi: I=\left[\wp_{1}, \wp_{2}\right] \subseteq \mathbb{R} \backslash\{0\} \longrightarrow \mathbb{R}$ be differentiable function on the $I^{\circ}$ of I. If $\psi^{\prime} \in L\left[\wp_{1}, \wp_{2}\right]$ and $\left|\psi^{\prime}\right|^{q}$ is an $m$-poly p-harmonic exp convex function on $I, q \geq 1$, then

$$
\begin{gathered}
\left|\frac{\psi\left(\wp_{1}\right)+\psi\left(\wp_{2}\right)}{2}-\frac{p \wp_{1}^{p} \wp_{2}^{p}}{\wp_{2}^{p}-\wp_{1}^{p}} \int_{\wp_{1}}^{\wp_{2}} \frac{\psi(x)}{x^{1+p}} d x\right| \leq \frac{\wp_{1} \wp_{2}\left(\wp_{2}^{p}-\wp_{1}^{p}\right)}{2 p} \\
\cdot\left\{G_{1}^{1-(1 / q)}\left[G_{2}\left|\psi^{\prime}\left(\wp_{1}\right)\right|^{q}+G_{3}\left|\psi^{\prime}\left(\wp_{2}\right)\right|^{q}\right]^{1 / q}\right\},
\end{gathered}
$$

where

$$
\begin{aligned}
G_{1} & =\int_{0}^{1} \frac{|1-2 \kappa|}{A_{\kappa}^{p+1}} d \kappa, G_{2}=\frac{1}{m} \int_{0}^{1} \frac{|1-2 \kappa| \sum_{j=1}^{m}\left(e^{\kappa}-1\right)^{j}}{A_{\kappa}^{1+p}} d \kappa, \\
G_{3} & =\frac{1}{m} \int_{0}^{1} \frac{|1-2 \kappa| \sum_{j=1}^{m}\left(e^{1-\kappa}-1\right)^{j}}{A_{\kappa}^{1+p}} d \kappa .
\end{aligned}
$$

Proof. Using Lemma 25, properties of modulus, power mean inequality, and $m$-poly $p$-harmonic exp convexity of the $\left|\psi^{\prime}\right|^{q}$, we have

$$
\begin{aligned}
& \left|\frac{\psi\left(\wp_{1}\right)+\psi\left(\wp_{2}\right)}{2}-\frac{p \wp_{1}^{p} \wp_{2}^{p}}{\wp_{2}^{p}-\wp_{1}^{p}} \int_{\wp_{1}}^{\mathfrak{\vartheta}_{2}} \frac{\psi(x)}{x^{1+p}} d x\right| \leq \frac{\wp_{1} \wp_{2}\left(\wp_{2}^{p}-\wp_{1}^{p}\right)}{2 p} \int_{0}^{1} \frac{|1-2 \kappa|}{A_{\kappa}^{p+1}} \\
& \quad \cdot\left|\psi^{\prime}\left(\frac{\wp_{1} \wp_{2}}{A_{\kappa}}\right)\right| d \kappa \leq \frac{\wp_{1} \wp_{2}\left(\wp_{2}^{p}-\wp_{1}^{p}\right)}{2 p}\left(\int_{0}^{1} \frac{|1-2 \kappa|}{A_{\kappa}^{p+1}} d \kappa\right)^{1-(1 / q)} \\
& \quad \cdot\left(\int_{0}^{1} \frac{|1-2 \kappa|}{A_{\kappa}^{p+1}}\left|\psi^{\prime}\left(\frac{\wp_{1} \wp_{2}}{A_{\kappa}}\right)\right|^{q} d \kappa\right)^{1 / q} \leq \frac{\wp_{1} \wp_{2}\left(\wp_{2}^{p}-\wp_{1}^{p}\right)}{2 p}\left(\int_{0}^{1} \frac{|1-2 \kappa|}{A_{\kappa}^{p+1}} d \kappa\right)^{1-(1 / q)} \\
& \times\left(\int_{0}^{1} \frac{|1-2 \kappa|\left[(1 / m) \sum_{j=1}^{m}\left(e^{\kappa}-1\right)^{j}\left|\psi^{\prime}\left(\wp_{1}\right)\right|^{q}+(1 / m) \sum_{j=1}^{m}\left(e^{1-\kappa}-1\right)^{j}\left|\psi^{\prime}\left(\wp_{2}\right)\right|^{q}\right]}{A_{\kappa}^{1+p}} d \kappa\right)^{1 / q} \\
& \leq \frac{\wp_{1} \wp_{2}\left(\wp_{2}^{p}-\wp_{1}^{p}\right)}{2 p}\left(\int_{0}^{1} \frac{|1-2 \kappa|}{A_{\kappa}^{p+1}} d \kappa\right)^{1-(1 / q)} \\
& \times\left(\frac{1}{m} \int_{0}^{1} \frac{|1-2 \kappa| \sum_{j=1}^{m}\left(e^{\kappa}-1\right)^{j}}{A_{\kappa}^{1+p}}\left|\psi^{\prime}\left(\wp_{1}\right)\right|^{q} d \kappa+\frac{1}{m} \int_{0}^{1} \frac{|1-2 \kappa| \sum_{j=1}^{m}\left(e^{1-\kappa}-1\right)^{j}}{A_{\kappa}^{1+p}}\left|\psi^{\prime}\left(\wp_{2}\right)\right|^{q} d \kappa\right)^{1 / q} \\
& \leq \frac{\wp_{1} \wp_{2}\left(\wp_{2}^{p}-\wp_{1}^{p}\right)}{2 p}\left\{G_{1}^{1-(1 / q)}\left[G_{2}\left|\psi^{\prime}\left(\wp_{1}\right)\right|^{q}+G_{3}\left|\psi^{\prime}\left(\wp_{2}\right)\right|^{q}\right]^{1 / q}\right\},
\end{aligned}
$$

which completes the proof.
Corollary 28. Under the assumptions of Theorem 27 with $p=-1$, we have the following new result:

$$
\begin{aligned}
& \left|\frac{\psi\left(\wp_{1}\right)+\psi\left(\wp_{2}\right)}{2}-\frac{1}{\wp_{2}-\wp_{1}} \int_{\wp_{1}}^{\wp_{2}} \psi(x) d x\right| \\
& \leq \frac{\left(\wp_{2}-\wp_{1}\right)}{2}\left(\frac{1}{2}\right)^{1-(1 / q)} \frac{1}{m} \sum_{j=1}^{m}\left(\frac{8 \sqrt{e}-2 e-7}{2}\right)^{j} \\
& \cdot\left\{\left[\left|\psi^{\prime}\left(\wp_{1}\right)\right|^{q}+\left|\psi^{\prime}\left(\wp_{2}\right)\right|^{q}\right]^{1 / q}\right\} .
\end{aligned}
$$

Corollary 29. Under the assumptions of Theorem 27 with $p=1$, we have the following new result:

$$
\begin{gathered}
\left|\frac{\psi\left(\wp_{1}\right)+\psi\left(\wp_{2}\right)}{2}-\frac{\wp_{1} \wp_{2}}{\wp_{2}-\wp_{1}} \int_{\wp_{1}}^{\wp_{2}} \frac{\psi(x)}{x^{2}} d x\right| \leq \frac{\wp_{1} \wp_{2}\left(\wp_{2}-\wp_{1}\right)}{2} \\
\cdot\left\{G_{1}{ }^{\prime 1-(1 / q)}\left[G_{2}^{\prime}\left|\psi^{\prime}\left(\wp_{1}\right)\right|^{q}+G_{3}^{\prime}\left|\psi^{\prime}\left(\wp_{2}\right)\right|^{q}\right]^{1 / q}\right\},
\end{gathered}
$$

where

$$
\begin{aligned}
& G_{1}^{\prime}=\int_{0}^{1} \frac{|1-2 \kappa|}{A_{\kappa}^{2}} d \kappa, G_{2}^{\prime}=\frac{1}{m} \int_{0}^{1} \frac{|1-2 \kappa| \sum_{j=1}^{m}\left(e^{\kappa}-1\right)^{j}}{A_{\kappa}^{2}} d \kappa, \\
& G_{3}^{\prime}=\frac{1}{m} \int_{0}^{1} \frac{|1-2 \kappa| \sum_{j=1}^{m}\left(e^{1-\kappa}-1\right)^{j}}{A_{\kappa}^{2}} d \kappa .
\end{aligned}
$$

Theorem 30. Let $\psi: I=\left[\wp_{1}, \wp_{2}\right] \subseteq \mathbb{R} \backslash\{0\} \longrightarrow \mathbb{R}$ be differentiable function on the $I^{\circ}$ of $I$. If $\psi^{\prime} \in L\left[\wp_{1}, \wp_{2}\right]$ and $\left|\psi^{\prime}\right|^{q}$ is an m-poly p-harmonic exp convex function on $I$, $r, q \geq 1,(1 / r)+(1 / q) \geq 1$, then,

$$
\begin{aligned}
& \left|\frac{\psi\left(\wp_{1}\right)+\psi\left(\wp_{2}\right)}{2}-\frac{p \wp_{1}^{p} \wp_{2}^{p}}{\wp_{2}^{p}-\wp_{1}^{p}} \int_{a}^{\wp_{2}} \frac{\psi(x)}{x^{1+p}} d x\right| \leq \frac{\wp_{1} \wp_{2}\left(\wp_{2}^{p}-\wp_{1}^{p}\right)}{2 p} \\
& \times\left\{G_{4}^{1 / r}\left[G_{5}\left|\psi^{\prime}\left(\wp_{1}\right)\right|^{q}+G_{6}\left|\psi^{\prime}\left(\wp_{2}\right)\right|^{q}\right]^{1 / q}\right\},
\end{aligned}
$$

where

$$
\begin{aligned}
& G_{4}=\int_{0}^{1}|1-2 \kappa|^{r} d \kappa, G_{5}=\frac{1}{m} \int_{0}^{1} \frac{\sum_{j=1}^{m}\left(e^{\kappa}-1\right)^{j}}{A_{\kappa}^{(1+p) q}} d \kappa, \\
& G_{6}=\frac{1}{m} \int_{0}^{1} \frac{\sum_{j=1}^{m}\left(e^{1-\kappa}-1\right)^{j}}{A_{\kappa}^{(1+p) q}} d \kappa .
\end{aligned}
$$

Proof. Using Lemma 25, properties of modulus, Hölder's inequality, and $m$-poly $p$-harmonic exp convexity of the $\left|\psi^{\prime}\right|^{q}$, we have 


$$
\begin{aligned}
& \left|\frac{\psi\left(\wp_{1}\right)+\psi\left(\wp_{2}\right)}{2}-\frac{p \wp_{1}^{p} \wp_{2}^{p}}{\wp_{2}^{p}-\wp_{1}^{p}} \int_{\wp_{1}}^{\wp_{2}} \frac{\psi(x)}{x^{1+p}} d x\right| \leq \frac{\wp_{1} \wp_{2}\left(\wp_{2}^{p}-\wp_{1}^{p}\right)}{2 p} \\
& \quad \cdot \int_{0}^{1} \frac{|1-2 \kappa|}{A_{\kappa}^{p+1}}\left|\psi^{\prime}\left(\frac{\wp_{1} \wp_{2}}{A_{\kappa}}\right)\right| d \kappa \leq \frac{\wp_{1} \wp_{2}\left(\wp_{2}^{p}-\wp_{1}^{p}\right)}{2 p} \\
& \quad \cdot\left(\int_{0}^{1}|1-2 \kappa|^{r} d \kappa\right)^{1 / r}\left(\int_{0}^{1} \frac{1}{\left.A_{\kappa}^{(1+p) q}\left|\psi^{\prime}\left(\frac{\wp_{1} \wp_{2}}{A_{\kappa}}\right)\right|^{q} d \kappa\right)^{1 / q}}\right. \\
& \leq \frac{\wp_{1} \wp_{2}\left(\wp_{2}^{p}-\wp_{1}^{p}\right)}{2 p}\left(\int_{0}^{1}|1-2 \kappa|^{r} d \kappa\right)^{1 / r} \\
& \times\left(\int_{0}^{1} \frac{1}{A_{\kappa}^{(1+p) q}}\left[\frac{1}{m} \sum_{j=1}^{m}\left(e^{\kappa}-1\right)^{j}\left|\psi^{\prime}\left(\wp_{1}\right)\right|^{q}+\frac{1}{m} \sum_{j=1}^{m}\left(e^{1-\kappa}-1\right)^{j}\left|\psi^{\prime}\left(\wp_{2}\right)\right|^{q}\right] d \kappa\right)^{1 / q} \\
& =\frac{\wp_{1} \wp_{2}\left(\wp_{2}^{p}-\wp_{1}^{p}\right)}{2 p}\left\{G_{4}^{1 / r}\left[G_{5}\left|\psi^{\prime}\left(\wp_{1}\right)\right|^{q}+G_{6}\left|\psi^{\prime}\left(\wp_{2}\right)\right|^{q}\right]^{1 / q}\right\},
\end{aligned}
$$

which completes the proof.

Corollary 31. Under the assumptions of Theorem 30 with $p=-1$, we have the following new result:

$$
\begin{aligned}
& \left|\frac{\psi\left(\wp_{1}\right)+\psi\left(\wp_{2}\right)}{2}-\frac{1}{\wp_{2}-\wp_{1}} \int_{\wp_{1}}^{\wp_{2}} \psi(x) d x\right| \\
& \leq \frac{\left(\wp_{2}-\wp_{1}\right)}{2}\left(\int_{0}^{1}|1-2 \kappa|^{r} d \kappa\right)^{1 / r} \frac{1}{m} \sum_{j=1}^{m}(e-2)^{j} \\
& \quad \cdot\left\{\left[\left|\psi^{\prime}\left(\wp_{1}\right)\right|^{q}+\left|\psi^{\prime}\left(\wp_{2}\right)\right|^{q}\right]^{1 / q}\right\} .
\end{aligned}
$$

Corollary 32. Under the assumptions of Theorem 30 with $p=1$, we have the following new result:

$$
\begin{gathered}
\left|\frac{\psi\left(\wp_{1}\right)+\psi\left(\wp_{2}\right)}{2}-\frac{\wp_{1} \wp_{2}}{\wp_{2}-\wp_{1}} \int_{\wp_{1}}^{\mathfrak{\wp}_{2}} \frac{\psi(x)}{x^{2}} d x\right| \leq \frac{\wp_{1} \wp_{2}\left(\wp_{2}-\wp_{1}\right)}{2} \\
\cdot\left\{G_{4}{ }^{11 / r}\left[G_{5}^{\prime}\left|\psi^{\prime}\left(\wp_{1}\right)\right|^{q}+G_{6}^{\prime}\left|\psi^{\prime}\left(\wp_{2}\right)\right|^{q}\right]^{1 / q}\right\},
\end{gathered}
$$

where

$$
\begin{aligned}
& G_{4}^{\prime}=\int_{0}^{1}|1-2 \kappa|^{r} d \kappa, G_{5}^{\prime}=\frac{1}{m} \int_{0}^{1} \frac{\sum_{j=1}^{m}\left(e^{\kappa}-1\right)^{j}}{A_{\kappa}^{2 q}} d \kappa, \\
& G_{6}^{\prime}=\frac{1}{m} \int_{0}^{1} \frac{\sum_{j=1}^{m}\left(e^{1-\kappa}-1\right)^{j}}{A_{\kappa}^{2 q}} d \kappa .
\end{aligned}
$$

Theorem 33. Let $\psi: I=\left[\wp_{1}, \wp_{2}\right] \subseteq \mathbb{R} \backslash\{0\} \longrightarrow \mathbb{R}$ be differentiable function on the $I^{\circ}$ of $I$. If $\psi^{\prime} \in L\left[\wp_{1}, \wp_{2}\right]$ and $\left|\psi^{\prime}\right|^{q}$ is an m-poly p-harmonic exp convex function on $I$, $q \geq, 1$ then

$$
\begin{aligned}
\mid \frac{1}{8} & {\left[\psi\left(\wp_{1}\right)+3 \psi\left(\left[\frac{3 \wp_{1}^{p} \wp_{2}^{p}}{\wp_{1}^{p}+2 \wp_{2}^{p}}\right]^{1 / p}\right)+3 \psi\left(\left[\frac{3 \wp_{1}^{p} \wp_{2}^{p}}{2 \wp_{1}^{p}+\wp_{2}^{p}}\right]^{1 / p}\right)+\psi\left(\wp_{2}\right)\right] } \\
& -\frac{p \wp_{1}^{p} \wp_{2}^{p}}{\wp_{2}^{p}-\wp_{1}^{p}} \int_{\wp_{1}}^{\wp_{2}} \frac{\psi(x)}{x^{1+p}} d x \mid \leq \frac{\wp_{1} \wp_{2}\left(\wp_{2}^{p}-\wp_{1}^{p}\right)}{p} \\
& \cdot\left\{B_{1}^{1-(1 / q)}\left[B_{4}\left|\psi^{\prime}\left(\wp_{1}\right)\right|^{q}+B_{5}\left|\psi^{\prime}\left(\wp_{2}\right)\right|^{q}\right]^{1 / q}\right. \\
& +B_{2}^{1-(1 / q)}\left[B_{6}\left|\psi^{\prime}\left(\wp_{1}\right)\right|^{q}+B_{7}\left|\psi^{\prime}\left(\wp_{2}\right)\right|^{q}\right]^{1 / q} \\
& \left.+B_{3}^{1-(1 / q)}\left[B_{8}\left|\psi^{\prime}\left(\wp_{1}\right)\right|^{q}+B_{9}\left|\psi^{\prime}\left(\wp_{2}\right)\right|^{q}\right]^{1 / q}\right\},
\end{aligned}
$$

where

$$
\begin{aligned}
& B_{1}=\int_{0}^{1 / 3} \frac{|\kappa-(1 / 8)|}{A_{\kappa}^{p+1}} d \kappa, B_{2}=\int_{1 / 3}^{2 / 3} \frac{|\kappa-(1 / 2)|}{A_{\kappa}^{p+1}} d \kappa, \\
& B_{4}=\frac{1}{m} \int_{0}^{1 / 3} \frac{|\kappa-(1 / 8)| \sum_{j=1}^{m}\left(e^{\kappa}-1\right)^{j}}{A_{\kappa}^{p+1}} d \kappa, \\
& B_{5}=\frac{1}{m} \int_{0}^{1 / 3} \frac{|\kappa-(1 / 8)| \sum_{j=1}^{m}\left(e^{1-\kappa}-1\right)^{j}}{A_{\kappa}^{p+1}} d \kappa, \\
& B_{6}=\frac{1}{m} \int_{1 / 3}^{2 / 3} \frac{|\kappa-(1 / 2)| \sum_{j=1}^{m}\left(e^{\kappa}-1\right)^{j}}{A_{\kappa}^{p+1}} d \kappa, \\
& B_{7}=\frac{1}{m} \int_{1 / 3}^{2 / 3} \frac{|\kappa-(1 / 2)| \sum_{j=1}^{m}\left(e^{1-\kappa}-1\right)^{j}}{A_{\kappa}^{p+1}} d \kappa, \\
& B_{8}=\frac{1}{m} \int_{2 / 3}^{1} \frac{|\kappa-(7 / 8)| \sum_{j=1}^{m}\left(e^{\kappa}-1\right)^{j}}{A_{\kappa}^{p+1}} d \kappa, \\
& B_{9}=\frac{1}{m} \int_{2 / 3}^{1} \frac{|\kappa-(7 / 8)| \sum_{j=1}^{m}\left(e^{1-\kappa}-1\right)^{j}}{A_{\kappa}^{p+1}} d \kappa .
\end{aligned}
$$

Proof. Using Lemma 26, properties of modulus, power mean inequality, and $m$-poly $p$-harmonic exp convexity of the $\left|\psi^{\prime}\right|^{q}$, we have

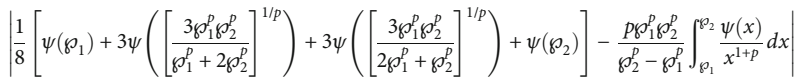

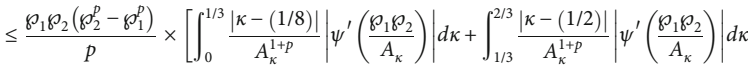

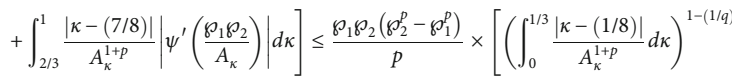

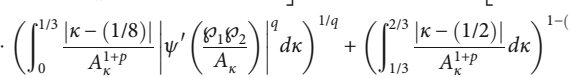

$$
\begin{aligned}
& \cdot\left(\int_{1 / 3}^{2 / 3} \frac{|\kappa-(1 / 2)|}{A_{\kappa}^{1+p}}\left|\psi^{\prime}\left(\frac{\mathcal{P}_{1} \vartheta_{2}}{A_{\kappa}}\right)\right|^{q} d \kappa\right)^{1 / q}+\left(\int_{2 / 3}^{1} \frac{|\kappa-(7 / 8)|}{A_{\kappa}^{1+p} d \kappa}\right)^{1-(1 / / q)}
\end{aligned}
$$

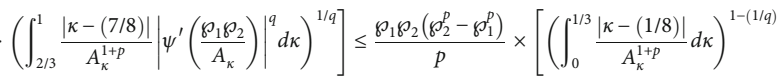

$$
\begin{aligned}
& \times\left(\int_{0}^{1 / 3} \frac{\kappa-(1 / 8) \mid\left[(1 / m) \sum_{j=1}^{m}\left(e^{\kappa}-1\right)^{j}\left|\psi^{\prime}\left(\mathfrak{p}_{1}\right)\right|^{q}+(1 / m) \sum_{j=1}^{m}\left(e^{1-\kappa}-1\right)^{j}\left|\psi^{\prime}\left(\boldsymbol{(}_{2}\right)\right|^{q}\right]}{A_{\kappa}^{1+p}} d \kappa\right)^{1 / q} \\
& +\left(\int_{1 / 3}^{2 / 3} \frac{|\kappa-(1 / 2)|}{A_{\kappa}^{1+p}} d \kappa\right)^{1-(1 / q)}
\end{aligned}
$$




$$
\begin{aligned}
& \times\left(\int_{1 / 3}^{2 / 3} \frac{|\kappa-(1 / 2)|\left[(1 / m) \sum_{j=1}^{m}\left(e^{\kappa}-1\right)^{j}\left|\psi^{\prime}\left(\wp_{1}\right)\right|^{q}+(1 / m) \sum_{j=1}^{m}\left(e^{1-\kappa}-1\right)^{j}\left|\psi^{\prime}\left(\wp_{2}\right)\right|^{q}\right]}{A_{\kappa}^{1+p}} d \kappa\right)^{1 / q} \\
& +\left(\int_{2 / 3}^{1} \frac{|\kappa-(7 / 8)|}{A_{\kappa}^{1+p}} d \kappa\right)^{1-(1 / q)} \\
& \left.\times\left(\int_{2 / 3}^{1} \frac{|\kappa-(7 / 8)|\left[(1 / m) \sum_{j=1}^{m}\left(e^{\kappa}-1\right)^{j}\left|\psi^{\prime}\left(\wp_{1}\right)\right|^{q}+(1 / m) \sum_{j=1}^{m}\left(e^{1-\kappa}-1\right)^{j}\left|\psi^{\prime}\left(\wp_{2}\right)\right|^{q}\right]}{A_{\kappa}^{1+p}} d \kappa\right)^{1 / q}\right] \\
& \leq \frac{\wp_{1} \wp_{2}\left(\wp_{2}^{p}-\wp_{1}^{p}\right)}{p} \times\left[\left(\int_{0}^{1 / 3} \frac{|\kappa-(1 / 8)|}{A_{\kappa}^{1+p}} d \kappa\right)^{1-(1 / q)} \times\left(\frac{1}{m} \int_{0}^{1 / 3} \frac{|\kappa-(1 / 8)| \sum_{j=1}^{m}\left(e^{\kappa}-1\right)^{j}}{A_{\kappa}^{1+p}}\right.\right. \\
& \left.\cdot\left|\psi^{\prime}\left(\wp_{1}\right)\right|^{q} d \kappa+\frac{1}{m} \int_{0}^{1 / 3} \frac{|\kappa-(1 / 8)| \sum_{j=1}^{m}\left(e^{1-\kappa}-1\right)^{j}}{A_{\kappa}^{1+p}}\left|\psi^{\prime}\left(\mathfrak{\wp}_{2}\right)\right|^{q} d \kappa\right)^{1 / q} \\
& +\left(\int_{1 / 3}^{2 / 3} \frac{|\kappa-(1 / 2)|}{A_{\kappa}^{1+p}} d \kappa\right)^{1-(1 / q)} \times\left(\frac{1}{m} \int_{1 / 3}^{2 / 3} \frac{|\kappa-(1 / 2)| \sum_{j=1}^{m}\left(e^{\kappa}-1\right)^{j}}{A_{\kappa}^{1+p}}\left|\psi^{\prime}\left(\mathfrak{\wp}_{1}\right)\right|^{q} d \kappa\right. \\
& \left.+\frac{1}{m} \int_{1 / 3}^{2 / 3} \frac{|\kappa-(1 / 2)| \sum_{j=1}^{m}\left(e^{1-\kappa}-1\right)^{j}}{A_{\kappa}^{1+p}}\left|\psi^{\prime}\left(\wp_{2}\right)\right|^{q} d \kappa\right)^{1 / q}+\left(\frac{1}{m} \int_{2 / 3}^{1} \frac{|\kappa-(7 / 8)|}{A_{t}^{1+p}} d t\right)^{1-(1 / q)} \\
& \times\left(\frac{1}{m} \int_{2 / 3}^{1} \frac{|\kappa-(7 / 8)| \sum_{j=1}^{m}\left(e^{\kappa}-1\right)^{j}}{A_{\kappa}^{1+p}}\left|\psi^{\prime}\left(\wp_{1}\right)\right|^{q} d \kappa+\frac{1}{m} \int_{2 / 3}^{1} \frac{|\kappa-(7 / 8)| \sum_{j=1}^{m}\left(e^{1-\kappa}-1\right)^{j}}{A_{\kappa}^{1+p}}\right.
\end{aligned}
$$

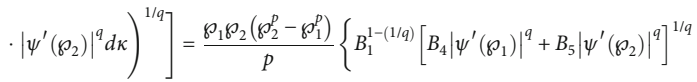

$$
\begin{aligned}
& \left.+B_{2}^{1-(1 / q)}\left[B_{6}\left|\psi^{\prime}\left(\mathfrak{\wp}_{1}\right)\right|^{q}+B_{7}\left|\psi^{\prime}\left(\mathfrak{\wp}_{2}\right)\right|^{q}\right]^{1 / q}+B_{3}^{1-(1 / q)}\left[B_{8}\left|\psi^{\prime}\left(\mathfrak{\wp}_{1}\right)\right|^{q}+B_{9}\left|\psi^{\prime}\left(\mathfrak{\wp}_{2}\right)\right|^{q}\right]^{1 / q}\right\},
\end{aligned}
$$

which completes the proof.

Corollary 34. Under the assumptions of Theorem 33 with $p=-1$ and $m=1$, we have the following new result:

$$
\begin{aligned}
\mid \frac{1}{8}\left[\psi\left(\wp_{1}\right)+3 \psi\left(\frac{2 \wp_{1}+\wp_{2}}{3}\right)+3 \psi\left(\frac{\wp_{1}+2 \wp_{2}}{3}\right)+\psi\left(\wp_{2}\right)\right] \\
-\frac{1}{\wp_{2}-\wp_{1}} \int_{\wp_{1}}^{\wp_{2}} \psi(x) d x \mid \leq\left(\wp_{2}-\wp_{1}\right) \\
\cdot\left\{\left(\frac{17}{576}\right)\left[0.0069\left|\psi^{\prime}\left(\wp_{1}\right)\right|^{q}+0.036\left|\psi^{\prime}\left(\wp_{2}\right)\right|^{q}\right]^{1 / q}\right. \\
+\left(\frac{0.183}{360}\right)\left[\left|\psi^{\prime}\left(\wp_{1}\right)\right|^{q}+\left|\psi^{\prime}\left(\wp_{2}\right)\right|^{q}\right]^{1 / q}+\left(\frac{17}{576}\right) \\
\left.\cdot\left[0.036\left|\psi^{\prime}\left(\wp_{1}\right)\right|^{q}+0.0069\left|\psi^{\prime}\left(\wp_{2}\right)\right|^{q}\right]^{1 / q}\right\} .
\end{aligned}
$$

Theorem 35. Let $\psi: I=\left[\wp_{1}, \wp_{2}\right] \subseteq \mathbb{R} \backslash\{0\} \longrightarrow \mathbb{R}$ be differentiable function on the $I^{\circ}$ of $I$. If $\psi^{\prime} \in L\left[\wp_{1}, \wp_{2}\right]$ and $\left|\psi^{\prime}\right|^{q}$ is an m-poly p-harmonic exp convex function on $I$, $r, q \geq 1$ and $(1 / r)+(1 / q) \geq 1$ then

$$
\begin{aligned}
\mid \frac{1}{8}\left[\psi\left(\wp_{1}\right)+3 \psi\left(\left[\frac{3 \wp_{1}^{p} \wp_{2}^{p}}{\wp_{1}^{p}+2 \wp_{2}^{p}}\right]^{1 / p}\right)+3 \psi\left(\left[\frac{3 \wp_{1}^{p} \wp_{2}^{p}}{2 \wp_{1}^{p}+\wp_{2}^{p}}\right]^{1 / p}\right)+\psi\left(\wp_{2}\right)\right] \\
\quad-\frac{p \wp_{1}^{p} \wp_{2}^{p}}{\wp_{2}^{p}-\wp_{1}^{p}} \int_{\wp_{1}}^{\wp_{2}} \frac{\psi(x)}{x^{1+p}} d x \mid \leq \frac{\wp_{1} \wp_{2}\left(\wp_{2}^{p}-\wp_{1}^{p}\right)}{p} \times\left\{\left(\frac{3^{r+1}+5^{r+1}}{24^{r+1}(r+1)}\right)^{1 / r}\right. \\
\cdot\left(B_{10}\left|\psi^{\prime}\left(\wp_{1}\right)\right|^{q}+B_{11}\left|\psi^{\prime}\left(\wp_{2}\right)\right|^{q}\right)^{1 / q}+\left(\frac{2}{6^{r+1}(r+1)}\right)^{1 / r} \\
\cdot\left(B_{12}\left|\psi^{\prime}\left(\wp_{1}\right)\right|^{q}+B_{13}\left|\psi^{\prime}\left(\wp_{2}\right)\right|^{q}\right)^{1 / q}+\left(\frac{3^{r+1}+5^{r+1}}{24^{r+1}(r+1)}\right)^{1 / r} \\
\left.\cdot\left(B_{14}\left|f^{\prime}(a)\right|^{q} d t+B_{15}\left|\psi^{\prime}\left(\wp_{2}\right)\right|^{q}\right)^{1 / q}\right\}
\end{aligned}
$$

where

$$
\begin{aligned}
B_{10} & =\frac{1}{m} \int_{0}^{1 / 3} \frac{\sum_{j=1}^{m}\left(e^{\kappa}-1\right)^{j}}{A_{\kappa}^{(1+p) q}} d \kappa, \\
B_{11} & =\frac{1}{m} \int_{0}^{1 / 3} \frac{\sum_{j=1}^{m}\left(e^{1-\kappa}-1\right)^{j}}{A_{\kappa}^{(1+p) q}} d \kappa, \\
B_{12} & =\frac{1}{m} \int_{1 / 3}^{2 / 3} \frac{\sum_{j=1}^{m}\left(e^{\kappa}-1\right)^{j}}{A_{\kappa}^{(1+p) q}} d \kappa, \\
B_{13} & =\frac{1}{m} \int_{1 / 3}^{2 / 3} \frac{\sum_{j=1}^{m}\left(e^{1-\kappa}-1\right)^{j}}{A_{\kappa}^{(1+p) q}} d \kappa, \\
B_{14} & =\frac{1}{m} \int_{2 / 3}^{1} \frac{\sum_{j=1}^{m}\left(e^{\kappa}-1\right)^{j}}{A_{\kappa}^{(1+p) q}} d \kappa, \\
B_{15} & =\frac{1}{m} \int_{2 / 3}^{1} \frac{\sum_{j=1}^{m}\left(e^{1-\kappa}-1\right)^{j}}{A_{\kappa}^{(1+p) q}} d \kappa .
\end{aligned}
$$

Proof. Using Lemma 26, properties of modulus, Hölder's inequality, and $m$-poly $p$-harmonic exp convexity of the $\left|\psi^{\prime}\right|^{q}$, we have

$$
\begin{aligned}
& \mid \frac{1}{8}\left[\psi\left(\wp_{1}\right)+3 \psi\left(\left[\frac{3 \wp_{1}^{p} \wp_{2}^{p}}{\wp_{1}^{p}+2 \wp_{2}^{p}}\right]^{1 / p}\right)+3 \psi\left(\left[\frac{3 \wp_{1}^{p} \wp_{2}^{p}}{2 \wp_{1}^{p}+\wp_{2}^{p}}\right]^{1 / p}\right)+\psi\left(\wp_{2}\right)\right] \\
& -\frac{p \wp_{1}^{p} \wp_{2}^{p}}{\wp_{2}^{p}-\wp_{1}^{p}} \int_{\wp_{1}}^{\wp_{2}} \frac{\psi(x)}{x^{1+p}} d x \mid \leq \frac{\wp_{1} \wp_{2}\left(\wp_{2}^{p}-\wp_{1}^{p}\right)}{p} \times\left[\int_{0}^{1 / 3}\left|\kappa-\frac{1}{8}\right|\right. \\
& \cdot\left|\frac{1}{A_{\kappa}^{1+p}} \psi^{\prime}\left(\frac{\wp_{1} \wp_{2}}{A_{\kappa}}\right)\right| d \kappa+\int_{1 / 3}^{2 / 3}\left|\kappa-\frac{1}{2}\right|\left|\frac{1}{A_{\kappa}^{1+p}} \psi^{\prime}\left(\frac{\wp_{1} \wp_{2}}{A_{\kappa}}\right)\right| d \kappa \\
& \left.+\int_{2 / 3}^{1}\left|\kappa-\frac{7}{8}\right|\left|\frac{1}{A_{\kappa}^{1+p}} \psi^{\prime}\left(\frac{\wp_{1} \wp_{2}}{A_{\kappa}}\right)\right| d \kappa\right] \leq \frac{\wp_{1} \wp_{2}\left(\wp_{2}^{p}-\wp_{1}^{p}\right)}{p} \\
& \left\{\left(\int_{0}^{1 / 3}\left|\kappa-\frac{1}{8}\right|^{r} d \kappa\right)^{1 / r}\left(\int_{0}^{1 / 3} \frac{1}{A_{\kappa}^{(1+p) q}}\left|\psi^{\prime}\left(\frac{\wp_{1} \wp_{2}}{A_{\kappa}}\right)\right|^{q} d \kappa\right)^{1 / q}\right. \\
& +\left(\int_{1 / 3}^{2 / 3}\left|\kappa-\frac{1}{2}\right|^{r} d \kappa\right)^{1 / r}\left(\int_{1 / 3}^{2 / 3} \frac{1}{A_{\kappa}^{(1+p) q}}\left|\psi^{\prime}\left(\frac{\wp_{1} \wp_{2}}{A_{\kappa}}\right)\right|^{q} d \kappa\right)^{1 / q} \\
& \left.+\left(\int_{2 / 3}^{1}\left|\kappa-\frac{7}{8}\right|^{r} d \kappa\right)^{1 / r}\left(\int_{2 / 3}^{1} \frac{1}{A_{\kappa}^{(1+p) q}}\left|\psi^{\prime}\left(\frac{\wp_{1} \wp_{2}}{A_{\kappa}}\right)\right|^{q} d \kappa\right)^{1 / q}\right\} \\
& \leq \frac{\wp_{1} \wp_{2}\left(\wp_{2}^{p}-\wp_{1}^{p}\right)}{p} \times\left\{\left(\int_{0}^{1 / 3}\left|\kappa-\frac{1}{8}\right|^{r} d \kappa\right)^{1 / r} \times\left(\int_{0}^{1 / 3} \frac{1}{A_{\kappa}^{(1+p) q}}\right.\right. \\
& \left.\cdot\left[\frac{1}{m} \sum_{j=1}^{m}\left(e^{\kappa}-1\right)^{j}\left|\psi^{\prime}\left(\wp_{1}\right)\right|^{q}+\frac{1}{m} \sum_{j=1}^{m}\left(e^{1-\kappa}-1\right)^{j}\left|\psi^{\prime}\left(\wp_{2}\right)\right|^{q}\right] d \kappa\right)^{1 / q} \\
& +\left(\int_{1 / 3}^{2 / 3}\left|\kappa-\frac{1}{2}\right|^{r} d \kappa\right)^{1 / r} \times\left(\int _ { 1 / 3 } ^ { 2 / 3 } \frac { 1 } { A _ { \kappa } ^ { ( 1 + p ) q } } \left[\frac{1}{m} \sum_{j=1}^{m}\left(e^{\kappa}-1\right)^{j}\left|\psi^{\prime}\left(\wp_{1}\right)\right|^{q}\right.\right. \\
& \left.\left.+\frac{1}{m} \sum_{j=1}^{m}\left(e^{1-\kappa}-1\right)^{j}\left|\psi^{\prime}\left(\wp_{2}\right)\right|^{q}\right] d \kappa\right)^{1 / q}+\left(\int_{2 / 3}^{1}\left|\kappa-\frac{7}{8}\right|^{r} d \kappa\right)^{1 / r} \\
& \left.\times\left(\int_{2 / 3}^{1} \frac{1}{A_{\kappa}^{(1+p) q}}\left[\frac{1}{m} \sum_{j=1}^{m}\left(e^{\kappa}-1\right)^{j}\left|\psi^{\prime}\left(\wp_{1}\right)\right|^{q}+\frac{1}{m} \sum_{j=1}^{m}\left(e^{1-\kappa}-1\right)^{j}\left|\psi^{\prime}\left(\wp_{2}\right)\right|^{q}\right] d \kappa\right)^{1 / q}\right\} \\
& =\frac{\wp_{1} \wp_{2}\left(\wp_{2}^{p}-\wp_{1}^{p}\right)}{p} \times\left\{( \frac { 3 ^ { r + 1 } + 5 ^ { r + 1 } } { 2 4 ^ { r + 1 } ( r + 1 ) } ) ^ { 1 / r } \left(\frac{1}{m} \int_{0}^{1 / 3} \frac{\sum_{j=1}^{m}\left(e^{\kappa}-1\right)^{j}}{A_{\kappa}^{(1+p) q}}\left|\psi^{\prime}\left(\wp_{1}\right)\right|^{q} d \kappa\right.\right. \\
& \left.+\frac{1}{m} \int_{0}^{1 / 3} \frac{\sum_{j=1}^{m}\left(e^{1-\kappa}-1\right)^{j}}{A_{\kappa}^{(1+p) q}}\left|\psi^{\prime}\left(\wp_{2}\right)\right|^{q} d \kappa\right)^{1 / q}+\left(\frac{2}{6^{r+1}(r+1)}\right)^{1 / r} \\
& \times\left(\frac{1}{m} \int_{1 / 3}^{2 / 3} \frac{\sum_{j=1}^{m}\left(e^{\kappa}-1\right)^{j}}{A_{\kappa}^{(1+p) q}}\left|\varphi^{\prime}\left(\wp_{1}\right)\right|^{q} d \kappa+\frac{1}{m} \int_{1 / 3}^{2 / 3} \frac{\sum_{j=1}^{m}\left(e^{1-\kappa}-1\right)^{j}}{A_{\kappa}^{(1+p) q}}\left|\psi^{\prime}\left(\wp_{2}\right)\right|^{q} d \kappa\right)^{1 / q}
\end{aligned}
$$




$$
\begin{aligned}
& +\left(\frac{3^{r+1}+5^{r+1}}{24^{r+1}(r+1)}\right)^{1 / r} \\
& \left.+\left(\frac{1}{m} \int_{2 / 3}^{1} \frac{\sum_{j=1}^{m}\left(e^{\kappa}-1\right)^{j}}{A_{\kappa}^{(1+p) q}}\left|\psi^{\prime}\left(\wp_{1}\right)\right|^{q} d \kappa+\frac{1}{m} \int_{2 / 3}^{1} \frac{\sum_{j=1}^{m}\left(e^{1-\kappa}-1\right)^{j}}{A_{\kappa}^{(1+p) q}}\left|\psi^{\prime}\left(\wp_{2}\right)\right|^{q} d \kappa\right)^{1 / q}\right\} \\
& =\frac{\wp_{1} \wp_{2}\left(\wp_{2}^{p}-\wp_{1}^{p}\right)}{p} \times\left\{\left(\frac{3^{r+1}+5^{r+1}}{24^{r+1}(r+1)}\right)^{1 / r}\left(B_{10}\left|\psi^{\prime}\left(\wp_{1}\right)\right|^{q}+B_{11}\left|\psi^{\prime}\left(\wp_{2}\right)\right|^{q}\right)^{1 / q}\right. \\
& +\left(\frac{2}{6^{r+1}(r+1)}\right)^{1 / r}\left(B_{12}\left|\psi^{\prime}\left(\wp_{1}\right)\right|^{q}+B_{13}\left|\psi^{\prime}\left(\wp_{2}\right)\right|^{q}\right)^{1 / q} \\
& \left.+\left(\frac{3^{r+1}+5^{r+1}}{24^{r+1}(r+1)}\right)^{1 / r}\left(B_{14}\left|\psi^{\prime}\left(\wp_{1}\right)\right|^{q} d \kappa+B_{15}\left|\psi^{\prime}\left(\wp_{2}\right)\right|^{q}\right)^{1 / q}\right\},
\end{aligned}
$$

which completes the proof.

Corollary 36. Under the assumptions of Theorem 35 with $p=-1$ and $m=1$, we have the following new result:

$$
\begin{aligned}
\mid \frac{1}{8}\left[\psi\left(\wp_{1}\right)+3 \psi\left(\frac{2 \wp_{1}+\wp_{2}}{3}\right)+3 \psi\left(\frac{\wp_{1}+2 \wp_{2}}{3}\right)+\psi\left(\wp_{2}\right)\right] \\
\quad-\frac{1}{\wp_{2}-\wp_{1}} \int_{\wp_{1}}^{\wp_{2}} \psi(x) d x \mid \leq\left(\wp_{2}-\wp_{1}\right)\left[\left(\frac{3^{r+1}+5^{r+1}}{24^{r+1}(r+1)}\right)^{1 / r}\right. \\
\quad \cdot\left(0.0623\left|\psi^{\prime}\left(\wp_{1}\right)\right|^{q}+0.4372\left|\psi^{\prime}\left(\wp_{2}\right)\right|^{q}\right)^{1 / q} \\
+\left(\frac{1}{6^{r+1}(r+1)}\right)^{1 / r} 0.2188\left(\left|\psi^{\prime}\left(\wp_{1}\right)\right|^{q}+\left|\psi^{\prime}\left(\wp_{2}\right)\right|^{q}\right)^{1 / q} \\
\left.\quad+\left(\frac{3^{r+1}+5^{r+1}}{24^{r+1}(r+1)}\right)^{1 / r}\left(0.4372\left|\psi^{\prime}\left(\wp_{1}\right)\right|^{q}+0.0623\left|\psi^{\prime}\left(\wp_{2}\right)\right|^{q}\right)^{1 / q}\right] .
\end{aligned}
$$

\section{Applications}

In this section, we recall the following special means of two positive number $\wp_{1}, \wp_{2}$ with $\wp_{1}<\wp_{2}$ :

(1) The arithmetic mean

$$
A=A\left(\wp_{1}, \wp_{2}\right)=\frac{\wp_{1}+\wp_{2}}{2}
$$

(2) The geometric mean

$$
G=G\left(\wp_{1}, \wp_{2}\right)=\sqrt{\wp_{1} \wp_{2}}
$$

(3) The harmonic mean

$$
H=H\left(\wp_{1}, \wp_{2}\right)=\frac{2 \wp_{1} \wp_{2}}{\wp_{1}+\wp_{2}}
$$

(4) The logarithmic mean

$$
L=L\left(\wp_{1}, \wp_{2}\right)=\frac{\wp_{2}-\wp_{1}}{\ln \wp_{2}-\ln \wp_{1}}
$$

These means have a lot of applications in areas and different types of numerical approximations. However, the following simple relationship are known in the literature:

$$
H\left(\wp_{1}, \wp_{2}\right) \leq G\left(\wp_{1}, \wp_{2}\right) \leq L\left(\wp_{1}, \wp_{2}\right) \leq A\left(\wp_{1}, \wp_{2}\right)
$$

Proposition 37. Let $0<\wp_{1}<\wp_{2}$ and $p \geq 1$. Then we get the following inequality

$$
\begin{aligned}
\frac{m}{2 \sum_{j=1}^{m}(\sqrt{e}-1)^{j}} H_{p}\left(\wp_{1}^{p}, \wp_{2}^{p}\right) & \leq \frac{p \wp_{1}^{p} \wp_{2}^{p}}{\wp_{2}^{p}-\wp_{1}^{p}}\left(\frac{\wp_{2}^{1-p}-\wp_{1}^{1-p}}{1-p}\right) \\
& \leq A\left(\wp_{1}, \wp_{2}\right) \frac{2}{m} \sum_{j=1}^{m}[e-2]^{j} .
\end{aligned}
$$

Proof. Taking $\psi(v)=v$ for $v>0$ in Theorem 21, then, inequality (59) is easily captured.

Proposition 38. Let $0<\wp_{1}<\wp_{2}$ and $p \geq 1$. Then, we get the following inequality:

$$
\begin{aligned}
\frac{m}{2 \sum_{j=1}^{m}(\sqrt{e}-1)^{j}} H_{2 p}^{-1}\left(\wp_{1}^{p}, \wp_{2}^{p}\right) & \leq \frac{p \wp_{1}^{p} \wp_{2}^{p}}{\wp_{2}^{p}-\wp_{1}^{p}}\left(\frac{\wp_{2}^{(1 / 2)-p}-\wp_{1}^{(1 / 2)-p}}{(1 / 2)-p}\right)^{-1} \\
& \leq A^{-1}\left(\sqrt{\wp}_{1}, \sqrt{\wp}_{2}\right) \frac{2}{m} \sum_{j=1}^{m}[e-2]^{j}
\end{aligned}
$$

Proof. Taking $\psi(v)=1 / \sqrt{v}$ for $v>0$ in Theorem 21, then, inequality (60) is easily captured.

Proposition 39. Let $0<\wp_{1}<\wp_{2}$ and $p \geq 1$. Then, we get the following inequality:

$$
\begin{aligned}
\frac{m}{2 \sum_{j=1}^{m}(\sqrt{e}-1)^{j}} H\left(\wp_{1}^{p}, \wp_{2}^{p}\right) & \leq \frac{p \wp_{1}^{p} \wp_{2}^{p}}{\wp_{2}^{p}-\wp_{1}^{p}}\left(\frac{\wp_{2}-\wp_{1}}{L\left(\wp_{1}, \wp_{2}\right)}\right) \\
& \leq A\left(\wp_{1}^{p}, \wp_{2}^{p}\right) \frac{2}{m} \sum_{j=1}^{m}[e-2]^{j} .
\end{aligned}
$$

Proof. Taking $\psi(v)=v^{p}$ for $v>0$ in Theorem 21, then, inequality (61) is easily captured.

Proposition 40. Let $0<\wp_{1}<\wp_{2}$ and $p \geq 1$. Then, we get the following inequality:

$$
\begin{aligned}
\frac{m}{2 \sum_{j=1}^{m}(\sqrt{e}-1)^{j}} H_{p}^{2}\left(\wp_{1}^{p}, \wp_{2}^{p}\right) & \leq \frac{p \wp_{1}^{p} \wp_{2}^{p}}{\wp_{2}^{p}-\wp_{1}^{p}}\left(\frac{\wp_{2}^{2-p}-\wp_{1}^{2-p}}{2-p}\right) \\
& \leq A\left(\wp_{1}^{2}, \wp_{2}^{2}\right) \frac{2}{m} \sum_{j=1}^{m}[e-2]^{j}
\end{aligned}
$$

Proof. Taking $\psi(v)=v^{2}$ for $v>0$ in Theorem 21, then, inequality (62) is easily captured. 
Proposition 41. Let $0<\wp_{1}<\wp_{2}$ and $p \geq 1$. Then, we get the following inequality:

$$
\begin{aligned}
\frac{m}{2 \sum_{j=1}^{m}(\sqrt{e}-1)^{j}} \ln G\left(\wp_{1}, \wp_{2}\right) & \leq \frac{p \wp_{1}^{p} \wp_{2}^{p}}{\wp_{2}^{p}-\wp_{1}^{p}} \int_{\wp_{1}}^{\wp_{2}} \frac{-\ln x}{x^{p+1}} d x \\
& \leq \ln H_{p}\left(\wp_{1}^{p}, \wp_{2}^{p}\right) \frac{2}{m} \sum_{j=1}^{m}[e-2]^{j} .
\end{aligned}
$$

Proof. Taking $\psi(v)=-\ln v$ for $v>0$ in Theorem 21, then, inequality (63) is easily captured.

Proposition 42. Let $0<\wp_{1}<\wp_{2}$. Then, we get the following inequality:

$$
\begin{aligned}
\frac{m}{2 \sum_{j=1}^{m}(\sqrt{e}-1)^{j}} e^{H\left(\mathfrak{\wp}_{1}, \mathfrak{\wp}_{2}\right)} & \leq \frac{p \wp_{1}^{p} \wp_{2}^{p}}{\wp_{2}^{p}-\wp_{1}^{p}} \int_{\wp_{1}}^{\wp_{2}} \frac{e^{x}}{x^{p+1}} d x \\
& \leq A\left(e^{\mathfrak{\wp}_{1}}, e^{\mathfrak{\wp}_{2}}\right) \frac{2}{m} \sum_{j=1}^{m}[e-2]^{j} .
\end{aligned}
$$

Proof. Taking $\psi(v)=e^{v}$ for $v>0$ in Theorem 21, then, inequality (64) is easily captured.

Proposition 43. Let $0<\wp_{1}<\wp_{2}$. Then, we get the following inequality:

$$
\begin{aligned}
A\left(\sin \wp_{1}, \sin \wp_{2}\right) \frac{2}{m} \sum_{j=1}^{m}[e-2]^{j} & \leq \frac{p \wp_{1}^{p} \wp_{2}^{p}}{\wp_{2}^{p}-\wp_{1}^{p}} \int_{\mathfrak{\wp}_{1}}^{\mathfrak{\wp}_{2}} \frac{\sin x}{x^{p+1}} d x \\
& \leq \frac{m}{2 \sum_{j=1}^{m}(\sqrt{e}-1)^{j}} \sin H_{p}\left(\wp_{1}, \wp_{2}\right) .
\end{aligned}
$$

Proof. Taking $\psi(v)=\sin (-v)$ for $v \in(0,(\pi / 2))$ in Theorem 21 , then, inequality (65) is easily captured.

Remark 44. The above discussed means, namely, arithmetic, geometric, harmonic, and logarithmic are well known in literature because these means have remarkable applications in machine learning, probability, statistics, and numerical approximation [24]. But, in the future, we will try to find the applications of the He Chengtian mean (also called as He Chengtian average), which was introduced by the first time a famous ancient Chinese mathematician He Chengtian [25]. The He Chengtian average was extended to solve nonlinear oscillators and it is called as He's max-min approach (also called as He's max-min method), which was further developed into a frequency-amplitude formulation for nonlinear oscillators $[26,27]$.

\section{Conclusion}

We have introduced and investigated some algebraic properties of a new class of functions, namely, $m$-poly $p$-harmonic exp convex. We showed that our new introduced class of function have some nice properties. We proved that our new introduced class is very larger with respect to the known class of functions, like $m$-polynomial convex and $m$-polynomial harmonically convex. A new version of Hermite-Hadamard type inequality and an integral identity for the differentiable function are obtained. It is high time to find the applications of these inequalities along with efficient numerical methods. We believe that our new class of functions will have a very deep research in this fascinating field of inequalities and also in pure and applied sciences. The interesting techniques and wonderful ideas of this paper can be extended on the coordinates along with fractional calculus. In the future, our goal is that we will continue our research work in this direction furthermore.

\section{Data Availability}

Data will be provided on request to the first author.

\section{Conflicts of Interest}

The authors declare that there are no conflicts of interest associated with this publication.

\section{Acknowledgments}

The authors received financial support from the Taif University Researches Supporting Project number (TURSP2020/031), Taif University, Taif, Saudi Arabia

\section{References}

[1] S. S. Dragomir and V. Pearce, Selected Topics on HermiteHadamard Inequalities and Applications, Victoria, RGMIA Monographs, 2000.

[2] K. Mehrez and P. Agarwal, "New Hermite-Hadamard type integral inequalities for convex functions and their applications," Journal of Computational and Applied Mathematics, vol. 350, pp. 274-285, 2019.

[3] D. S. Mitrinović, J. E. Pečarić, and A. M. Fink, Classical and new inequalities in analysis. Mathematics and its Applications (East European Series), vol. 61, Kluwer Academic Publishers Group, Dordrecht, 1993.

[4] Y. Khurshid, M. Adil Khan, and Y.-M. Chu, "Conformable integral inequalities of the Hermite-Hadamard type in terms of GG- and GA-convexities," Journal of Function Spaces, vol. 2019, Article ID 6926107, 8 pages, 2019.

[5] C. P. Niculescu and L. E. Persson, Convex Functions and Their Applications, Springer, New York, 2006.

[6] S. Varošanec, "On h-convexity," Journal of Mathematical Analysis and Applications, vol. 326, pp. 303-311, 2007.

[7] T. Toplu, M. Kadakal, and İ.İşcan, "On $n$-polynomial convexity and some related inequalities," AIMS Mathematics, vol. 5, no. 2, pp. 1304-1318, 2020.

[8] H. N. Shi and J. Zhang, "Some new judgement theorems of Schur geometric and schur harmonic convexities for a class of symmetric function," Journal of Inequalities and Applications, vol. 2013, Article ID 527, 2013.

[9] G. D. Anderson, M. K. Vamanamurthy, and M. Vuorinen, "Generalized convexity and inequalities," Journal of 
Mathematical Analysis and Applications, vol. 335, no. 2, pp. 1294-1308, 2007.

[10] M. A. Noor, K. I. Noor, and S. Iftikhar, "Harmite-Hadamard inequalities for harmonic nonconvex function," MAGNT Research Report, vol. 4, no. 1, pp. 24-40, 2016.

[11] M. U. Awan, N. Akhtar, S. Iftikhar, M. A. Noor, and Y.M. Chu, "New Hermite-Hadamard type inequalities for npolynomial harmonically convex functions," Journal of Inequalities and Applications, vol. 2020, Article ID 125, 2020.

[12] S. I. Butt, A. Kashuri, M. Tariq, J. Nasir, A. Aslam, and W. Gao, " $n$-polynomial exponential type p-convex function with some related inequalities and their applications," Heliyon, vol. 6, article e05420, 2020.

[13] S. I. Butt, A. Kashuri, M. Tariq, J. Nasir, A. Aslam, and W. Gao, "Hermite-Hadamard-type inequalities via n-polynomial exponential-type convexity and their applications," Advances in Difference Equations, vol. 2020, Article ID 508, 2020.

[14] S. S. Dragomir and I. Gomm, "Some Hermite-Hadamard's inequality functions whose exponentials are convex," Babes Bolyai Math., vol. 60, pp. 527-534, 2015.

[15] M. U. Awan, N. Akhtar, S. Iftikhar, M. A. Noor, and Y. -M. Chu, "Hermite-Hadamard type inequalities for exponentially convex functions," Applied Mathematics \& Information Sciences, vol. 12, no. 2, pp. 405-409, 2018.

[16] M. Kadakal and İ. İşcan, "Exponential type convexity and some related inequalities," Journal of Inequalities and Applications, vol. 2020, Article ID 82, 2020.

[17] W. Geo, A. Kashuri, S. I. Butt, M. Tariq, A. Aslam, and M. Nadeem, "New inequalities via $n$-polynomial harmoniaclly exponential type convex functions," AIMS Mathematics, vol. 5, no. 6, pp. 6856-6873, 2020.

[18] G. Alirezaei and R. Mahar, "On exponentially concave functions and their impact in information theory," in 2018 Information Theory and Applications Workshop (ITA), pp. 1-10, San Diego, CA, USA, 2018.

[19] S. Pal and T. K. L. Wong, "Exponentially concave functions and a new information geometry," The Annals of Probability, vol. 46, no. 2, pp. 1070-1113, 2018.

[20] İ. İşcan, "Hermite-Hadamard type inequalities for harmonically convex functions," Hacettepe Journal of Mathematics, vol. 43, no. 6, pp. 935-942, 2014.

[21] M. A. Noor, K. I. Noor, and S. Iftikhar, "Integral inequalities for diffrential p-harmonic convex function," Filomet, vol. 31, no. 20, pp. 6575-6584, 2017.

[22] M. A. Noor, K. I. Noor, M. U. Awan, and S. Costache, "Some integral inequalities for harmonically h-convex functions," UPB Scientific Bulletin, Series A, vol. 77, no. 1, pp. 5-16, 2015.

[23] M. A. Noor, K. I. Noor, and S. Iftikhar, "Newton inequalities for $p$-harmonic convex function," Honam Mathematical Journal, vol. 40, no. 2, pp. 239-250, 2018.

[24] R. J. Dalpatadu, The Arithmetic-Geometric-Harmonic Mean, JSM Math. Stat., 2014.

[25] J. H. He, "He Chengtian's inequality and its applications," Applied Mathematics and Computation, vol. 151, no. 3, pp. 887-891, 2004.
[26] J. H. He, "Max-min approach to nonlinear oscillators," International Journal of Nonlinear Sciences and Numerical Simulation, vol. 9, no. 2, pp. 207-210, 2008.

[27] J. H. He, "An improved amplitude-frequency formulation for nonlinear oscillators," International Journal of Nonlinear Sciences and Numerical Simulation, vol. 9, no. 2, pp. 211-212, 2008. 